\title{
Measurement Method for Non-circular Piston Based on Laser Displacement Sensor
}

\author{
Yanwei $X U^{a}$, Tancheng $\mathrm{XIE}^{\mathrm{b}}$, Junpeng $\mathrm{WANG}^{\mathrm{c}}$ and Menghui $\mathrm{CHEN}^{\mathrm{d}}$ \\ School of Mechatronics Engineering, Henan University of Science and Technology, Luoyang, \\ 471003, China \\ axuyanweiluoyang@163.com, ${ }^{\mathrm{b}}$ xietc@mail.haust.edu.cn, ${ }^{\mathrm{c}}$ wangjunpeng@163.com,
}

Keywords: Metrology, Non-circular piston, Laser displacement sensor, Measurement.

Abstract. A method of on-line measurement which is based on a loser displacement sensor to measure external profile of non-circular piston is proposed. Measurement coordinate system is established on the basis of the reference cylinder. Measurement data is processed through least square method to achieve the actual value of piston-section. Compared the actual value with the designed value can determine whether the cross section of piston is qualified. Fitting spatial line which is consisted of a plurality of cross-sectional center forms the axis of the elliptic cylinder. Then we can obtain straightness of the piston neutral line. The method of on-line measurement is validated through making specific experiment. This method is available to evaluate processing quality of external contour. At the same time, it is also helpful to make on-line processing correction for piston.

\section{Introduction}

This paper presents a non-contact online measurement method for non-circular piston, which allows the piston directly detecting without dismantling after CNC lathe. The method does not exist to re-locate the problem, collect data with a laser displacement sensor, and then the data to the host computer for processing piston to complete assessment of processing quality. Not only for the complete cross-section of the piston head were evaluated, but also complement incomplete oval cross-section data exists pin hole located at piston skirt. Fitting the axis of the piston, assessed shape and position accuracy of the piston with the axis of straightness, thus completing the whole of the piston outer contour measurement and evaluation. It has application value in terms of piston machining error correction online.

\section{Online measurement theory}

Piston outer contour is more complex, different elliptical cross-sectional shape of different heights, and the piston head is complete elliptical cross-section and the piston skirt due to the presence of pin holes whose cross-sectional shape is incomplete elliptic. In order to better piston-line measurement and evaluation, a mathematical model of the piston cross-section of line detection is established on the basis of studying the ellipse fitting.

\section{Establish measurement coordinate system}

In order to facilitate data processing, established a measurement coordinate system in the plane where the cross section of the piston is necessary [1]. Chucking a radius $r$ of the reference cylinder in $\mathrm{CNC}$ lathes, adjusting the laser displacement sensor parallel to the axis $\mathrm{CNC}$ lathes, measured distance from cylinder to the sensor is $L_{0}$. With lathe axis as a rotation axis measurements, establish measurement coordinate system in the plane perpendicular to the axis of rotation [2]. Pistons measurement with a laser displacement sensor in the same location after the machining, the measured distance from sensors to the piston is $L_{i}$. The distance from point of the piston on the contour to the axis of rotation is $p_{i}=L_{0}+r-L_{i}$. Set $\left(x_{i}, y_{i}\right)$ is the coordinates of the point on the ellipse piston contour, 
then $x_{i}=p_{i} \cos \theta_{i}, y_{i}=p_{i} \sin \theta_{i}$.In the formula, $\theta_{i}$ is the angle between $p_{i}$ and $x$ axes. The online measurement coordinate system is shown in Fig.1.

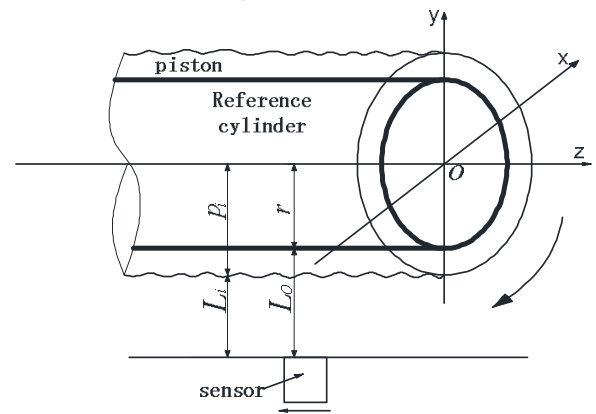

Fig.1 Establish an online measurement coordinate system

\section{Evaluation elliptical cross-sectional profile}

As a result of the existence of the piston processing error, the actual cross-sectional profile can be regarded as a closed curve based on ellipse, its center may not coincide with the theoretical design origin, so the data processing is necessary。Seeking real oval center and the polar radius of piston cross section by using the least squares method to fit the elliptic equations, and then obtained elliptical cross section profile error by compared with the design size. The elliptical cross section of the piston online measurement schematics is shown in Fig.2.

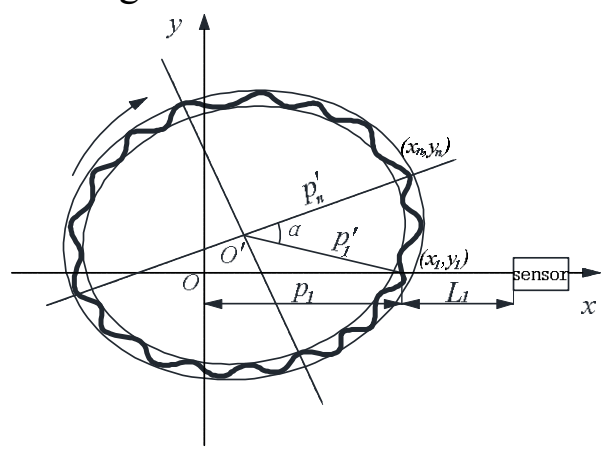

Fig.2 Elliptical cross section of the piston online measurement schematics

\section{Sectional profile of the piston skirt assessment}

Due to the presence of the piston pin hole, the skirt portion elliptical cross-sectional profile of the measured data is incomplete, we cannot directly use the method of least squares fitting ellipse. It is generally only the long axis of the skirt portion size was measured. In this paper, we complete missing parameter points with a theoretical value, and then use the elliptical cross-sectional profile of the head of evaluation methods for data processing, get profile error of the piston skirt portion of the oval cross-section. The section of the piston skirt finished up figure is shown in Fig.3.

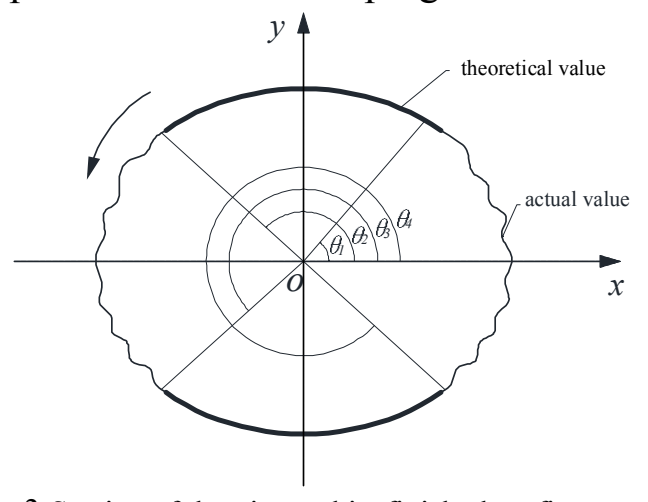

Fig.3 Section of the piston skirt finished up figure

Setting $\theta_{1} 、 \theta_{3}$ is the Starting position of missing points, $\theta_{2} 、 \theta_{4}$ is the end position of missing points. The expressions with the design value to complement the missing polar radius parameter is: 


$$
p=\frac{a b}{\sqrt{a^{2} \sin ^{2} \theta+b^{2} \cos ^{2} \theta}} \quad \theta \in\left(\theta_{1}, \theta_{3}\right) \cup\left(\theta_{2}, \theta_{4}\right)
$$

\section{Piston center line straightness}

In the piston parts drawing, position accuracy of the piston axis (verticality and the full end face beating) is also required, but because of the different parameters of the elliptical cross section of the piston at different heights, so difficult to measure the axis position tolerance. This article uses the axis straightness to online assess the axis of the piston shape and position accuracy. From the piston section contour assess we known the center of sectional profile. After a plurality of elliptical cross section measured we get a plurality of cross-sectional center data $\left(x_{i}, \mathrm{y}_{i}, z_{i}\right)(i=0,1, \cdots n)$. With these points fit piston centerline equation, the maximum distance from elliptical cross section of the center to the center line is straightness centerline [3]. Straightness of the piston centerline is shown in Fig.4.

Standard linear equation for space is:

$$
\frac{x-x_{0}}{k_{1}}=\frac{y-y_{0}}{k_{2}}=\frac{z-z_{0}}{k_{3}}
$$

Simplification available:

$$
\left\{\begin{array}{l}
x=\frac{k_{1}}{k_{2}}\left(z-z_{0}\right)+x_{0}=a z+b \\
y=\frac{k_{2}}{k_{3}}\left(z-z_{0}\right)+y_{0}=c z+d
\end{array}\right.
$$

Space linear equation is obtained, then the distance between ellipse center and the fitting line space is:

$$
D_{i}=\frac{\sqrt{\left(-y_{i}+c z_{i}+d\right)^{2}+\left(x_{i}-a z_{i}-b\right)^{2}+\left(-c x_{i}+a y_{i}+b c-a d\right)^{2}}}{\sqrt{a^{2}+c^{2}+1}}
$$

So the straightness error of piston centerline is:

$$
f_{M Z}=2 D_{\max }
$$

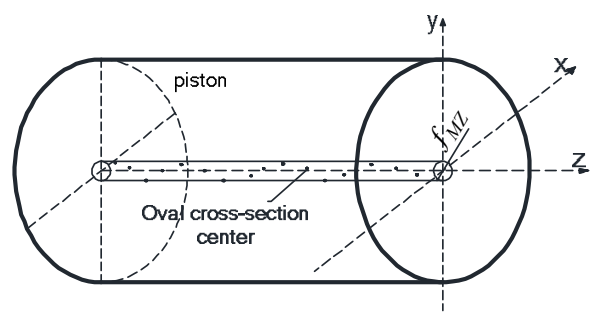

Fig.4 Straightness of the piston centerline

\section{Experimental verification}

In this paper, Isuzu engine piston use this method to measure. From the piston part drawing we can know the first outer diameter of the piston ring shore is $d=85.78_{-0.05}^{0} \mathrm{~mm}$, ellipticity is $e=0.08$, the semi-major axis's length $a=42.89_{-0.05}^{0} \mathrm{~mm}$, the semi-minor axis's length $b=42.85_{-0.05}^{0} \mathrm{~mm}$. The Isuzu piston line detection is shown in Fig.5. 


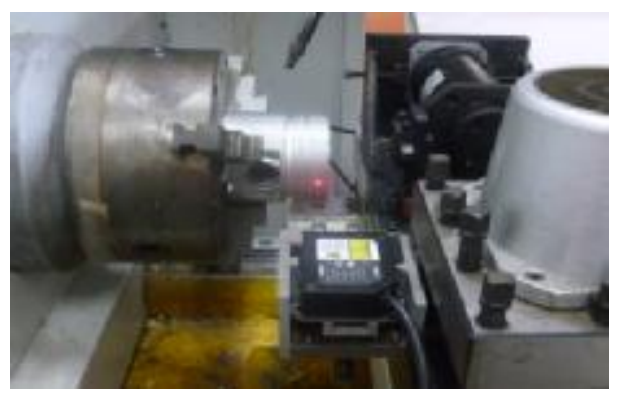

Fig.5 Isuzu piston line detection

Measuring the second section of the piston ring shore, get 60 points measured values. Compared with the theoretical value, the results is shown in Fig.6.

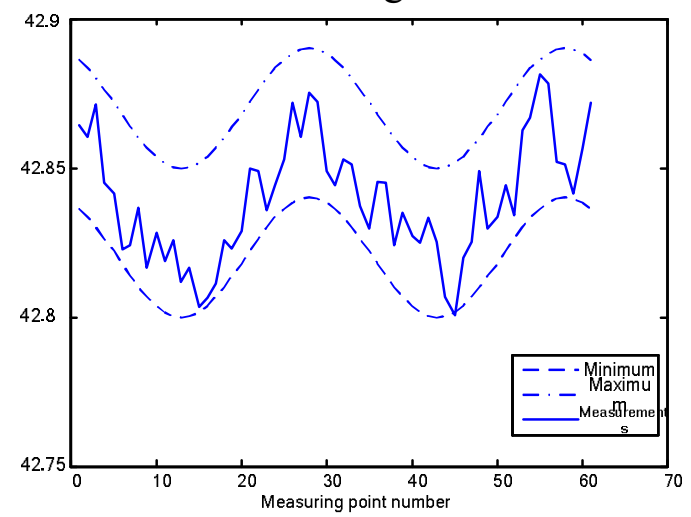

Fig. 6 Isuzu piston head measurements

Isuzu piston skirt located at the height of $16 \mathrm{~mm}$, the long axis dimension is $d=86_{-0.03}^{-0.01} \mathrm{~mm}$, ellipticity is $e=0.36$, the semi-major axis's length $a=43_{-0.03}^{-0.01} \mathrm{~mm}$, the semi-minor axis's length $b=42.82_{-0.03}^{-0.01} \mathrm{~mm}$. With the design value of the piston skirt complete data of piston section, then the piston skirt section to be assessed. The measurement results is shown in Fig.7.

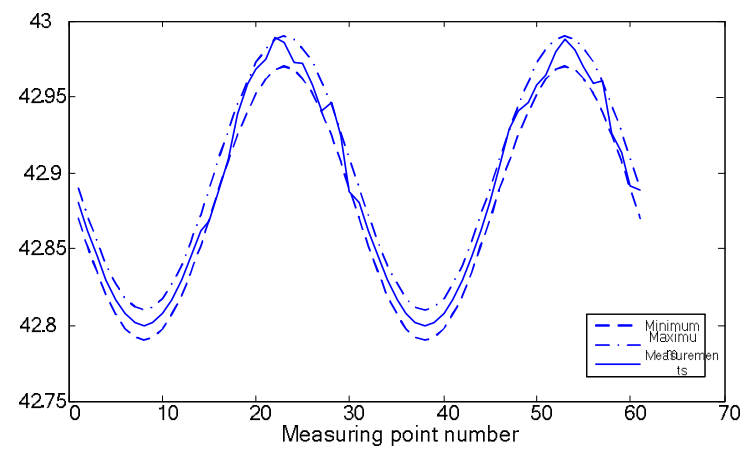

Fig. 7 Measurement results Isuzu piston skirt

Measuring Isuzu piston at the height of $4,16,32,48,58,64 \mathrm{~mm}$ cross-section to give a cross-sectional of the ellipse of least squares : $\left(1.0664,0.1969 \times 10^{-14}\right) 、\left(1.0666,0.0711 \times 10^{-14}\right)$ 、 $\left(1.0656,-0.0283 \times 10^{-14}\right) 、\left(1.0653,0.2133 \times 10^{-14}\right) 、\left(1.0627,-0.0333 \times 10^{-14}\right) 、\left(1.0634,-0.0383 \times 10^{-14}\right)$, Piston center line from these points fitting is shown in Fig.8. 


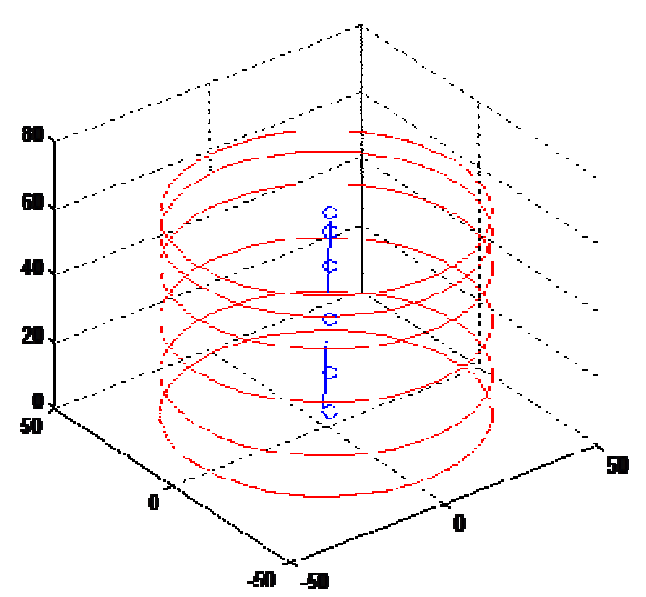

Fig. 8 Isuzu piston center line straightness

At the height of $16 \mathrm{~mm}$ cross-section, the maximum distance from the least-square circle center to the fitting centerline is $0.000057 \mathrm{~mm}$.By equation (17), Isuzu piston center line straightness is $0.000114 \mathrm{~mm}$.

\section{Conclusion}

(1) Laser displacement sensor is used to establish the non-circular piston online measurement of measuring coordinate system, make the piston online data acquisition becomes a reality.

(2) According to the principle of least square method, the establishment of the mathematical model of elliptical cross section contour, finding the measured values compared with design value for piston profile error of elliptic cross section. With the theoretical value complement piston skirt incomplete oval cross-section parameters.

(3) Using multiple sectional ellipse center fitting the center line of the piston, evaluating the piston axis position tolerance.

(4) Measurement method of experimental verification results show that the method can meet the requirements of precision measurement piston. It can effectively solve the problem of online testing of non-circular cross-section of the piston, to provide technical support for further detection of high-precision closed-loop processing piston.

\section{Acknowledgements}

This work was financially supported by the National Natural Science Foundation of China (51305127) and the scientific research key project fund of the Education Department Henan Province of China (14A460018).

\section{References}

[1] Daocheng Yuan,Yang WANG. Laser displacement sensor suitable for on-line measurement [J], Measurement Technology, 2002(5):3-6.

[2] Qifang $\mathrm{Lu}$. Measuring circle with optoelectronic and non-contact based on laser displacement sensor[J]. Electronic Design Engineering, 2011, 19(1):106-108.

[3] Lili Xing,Shuaiyun Zhang,Fusheng Jing. Piston-type wire clamp detection accuracy analysis[J]. Techinque Solutions, 2014, (23):50-51. 